\title{
RELAÇÃO UMIDADE VERSUS RESISTÊNCIA À PENETRAÇÃO PARA UM ARGISSOLO AMARELO DISTROCOESO NO RECÔNCAVO DA BAHIA ${ }^{(1)}$
}

\author{
Dreid de Cerqueira Silveira ${ }^{(2)}$, José Fernandes de Melo Filho ${ }^{(3)}$, José Augusto \\ Amorim Silva do Sacramento ${ }^{(4)} \&$ Edvana Cássia Pinto Silveira ${ }^{(5)}$
}

\begin{abstract}
RESUMO
A resistência do solo à penetração é considerada a propriedade mais adequada para expressar o grau de compactação do solo e, consequentemente, a facilidade que este oferece à penetração das raízes. Por isso, sua quantificação representa um importante indicativo da dinâmica de crescimento e desenvolvimento do sistema radicular das plantas. $\mathrm{O}$ objetivo deste trabalho foi caracterizar a relação entre a umidade do solo e a resistência à penetração em um Argissolo Amarelo distrocoeso na região do Recôncavo da Bahia. O estudo foi realizado no município de Governador Mangabeira - BA, em um pomar comercial de laranja 'Pêra' (Citrus sinensis Osb.) sob porta-enxerto de limão-cravo (Citrus limonia Osbeck) cultivado com feijão-de-porco (Canavalia ensiformis L.). As avaliações foram realizadas entre junho e dezembro de 2008, com um penetrômetro de impacto modelo Stoff (1991), e a amostragem do solo, em duas profundidades: 0,0-0,20 e 0,20-0,40 m, nas entrelinhas da cultura. Para caracterização da relação umidade do solo versus resistência à penetração de raízes, realizou-se a avaliação estatística descritiva e testaram-se quatro modelos de ajuste: dois exponenciais e dois potenciais. Os resultados demonstram que a resistência à penetração do solo apresentou distribuição diferente da normal e elevado coeficiente de variação, assim como os modelos matemáticos testados mostraram bom ajuste; o modelo exponencial foi o que apresentou os melhores índices de aferição para a relação umidade do solo versus resistência à penetração.
\end{abstract}

Termos de indexação: penetrômetro, qualidade do solo, citros, solos coesos.

\footnotetext{
(1) Recebido para publicação em agosto de 2009 e aprovado em março de 2010.

(2) Mestranda do Curso de Pós-Graduação em Ciências Agrárias - Ciência do Solo do CCAAB, Universidade Federal do Recôncavo da Bahia - UFRB. CEP 44380-000 Cruz das Almas (BA). Bolsista CNPq. E-mail: dreidcalsinal@hotmail.com

(3) Professor Adjunto do Centro de Ciências Agrárias, Ambientais e Biológicas, UFRB. Bolsista MEC/PET E-mail: jfmelo@ufrb.edu.br

(4) Graduando em Agronomia do CCAAB, UFRB. Bolsista PIBIC CNPq. E-mail: jassacramento@yahoo.com.br

(5) Engenheira-Agrônoma. Mestre em Ciências Agrárias. E-mail: edvanapinto@yahoo.com.br
} 


\title{
SUMMARY: RELATIONSHIP BETWEEN THE SOIL WATER CONTENT AND ROOT PENETRATION RESISTANCE OF A DYSTROCOHESIVE YELLOW ARGISSOL
}

\begin{abstract}
Soil resistance to root penetration is the main parameter to express the soil compaction degree and the facilities that it offers to root penetration. The purpose of this study was to characterize the ratio of the soil water content and root penetration resistance of a DystrocohesiveYellow Argissol in the Recôncavo Baiano (Bahia, Brazil). The study was carried out in a commercial citrus orchard with 'Pera'sweet orange (Citrus sinensis Osb.) grafted on 'Rangpur' lime (Citrus limonia Osbeck) with a green cover of jack beans (Canavalia ensiformes, L.) in Governador Mangabeira county. The soil resistance to root penetration was determined using the impact penetrometer at depths of 0.0-0.20 m;0.20-0.40 m, in between the planting lines of citrus, from June to December 2008. To characterize the relationship between the soil water content and the soil resistance to root penetration a descriptive statistical evaluation was performed by fitting four models; two potential and two exponential. Results showed that the distribution of the root penetration resistance of the Distrocohesive Yellow Argissol was nonnormal and the coefficient of variation was high. The models tested fitted well and are adequate to describe the relationship between the soil water content and soil resistance to root penetration; the fitting of the exponential model to the data was better than that observed for the potential model.
\end{abstract}

Index terms: penetrometer, soil quality, citrus, cohesive soils.

\section{INTRODUÇÃO}

As condições físicas do solo alteram direta e indiretamente a produção vegetal e a qualidade ambiental. Por isso, solos com bom índice de agregação e bem manejados funcionam adequadamente para manter o balanço equilibrado de ar e água, a ciclagem de nutrientes e o crescimento do sistema radicular das plantas (Arshad et al., 1996; Reynolds et al., 2002). Essa é uma condição dinâmica, dependente das características pedogenéticas do solo e fortemente influenciada pelas condições de uso e manejo (Tormena et al., 2002). Para a qualidade física, a resistência do solo à penetração é considerada a propriedade mais adequada para expressar o grau de compactação do solo e, consequentemente, a facilidade de penetração das raízes. Por isso, sua quantificação representa importante indicativo da dinâmica de crescimento e desenvolvimento do sistema radicular das plantas (Stolf et al., 1983; Tormena \& Roloff, 1996; Pereira et al., 2002; Mercante et al., 2003).

Segundo Pedrotti et al. (2001), a resistência à penetração serve para descrever a resistência física que o solo oferece a algo que tenta se mover através dele. Ela está diretamente correlacionada com vários atributos e condição do solo, como textura, densidade, matéria orgânica e, principalmente, a umidade $(\theta)$ no momento da determinação (Stolf et al., 1983; Busscher et al., 1997). Também pode ser influenciada pela coesão (Giarola et al., 2001).

A coesão é um atributo diagnóstico utilizado no Sistema Brasileiro de Classificação de Solos para descriminar Latossolos Amarelos com horizontes pedogenéticos subsuperficiais, $\mathrm{AB}$ e, ou, $\mathrm{BA}$, entre 0,3 e $0,7 \mathrm{~m}$ da superfície do solo, adensados, muito duros a extremamente duros quando secos, passando a friáveis ou firmes quando úmidos (Embrapa, 2006). Suas principais características são a densidade alta, baixa permeabilidade e elevada resistência à penetração quando secos (Cintra et al., 1997; Giarola et al., 2001; Libardi \& Melo Filho, 2001). Em consequência dessas características, o horizonte coeso funciona como uma forte limitação para a produção agrícola (Rezende, 2000). Nessas condições, o sistema radicular das plantas sofre modificações morfológicas e fisiológicas, as quais dependem da espécie ou cultivar (Muller et al., 2001). Para o citros, Cintra et al. (1999), Santana (2003) e Souza et al. (2008) observaram limitações ao crescimento das raízes em profundidade, e Carvalho et al. (2001), efeitos adversos na produtividade e na longevidade das plantas.

Em razão dessa diversidade de relações e influências, diversos modelos matemáticos foram testados para descrever a relação entre os atributos do solo e a resistência à penetração de raízes, dentre os quais aqueles definidos pela densidade e umidade do solo são os principais (Busscher et al., 1997; Pereira et al., 2002; Patrizzi et al., 2003). Essas relações já estão bem descritas na literatura para as mais diversas condições de solos e sistemas de uso e manejo (Tormena \& Roloff, 1996; Busscher et al., 1997; Fernandes et al., 2007). Por outro lado, esses atributos, pela facilidade de determinação e forte correlação, são utilizados para realizar inferências sobre as condições físicas do solo em relação aos valores críticos de 
resistência mecânica ao crescimento das raízes, possibilitando a adoção de estratégias de manejo para minimizar esses efeitos (Imhoff et al., 2000), sendo, por isso, uma importante ferramenta de aplicação prática.

Nesse contexto, este trabalho teve como objetivo caracterizar a relação entre a umidade do solo e a resistência à penetração em um Argissolo Amarelo distrocoeso na região do Recôncavo da Bahia.

\section{MATERIAL E MÉTODOS}

\section{Descrição da área e solo}

O estudo foi realizado no período de abril a dezembro de 2008, em um pomar comercial de citros localizado no município de Governador Mangabeira, região do Recôncavo do Estado da Bahia, cujas coordenadas geográficas são: $12^{\circ} 36^{\prime} 00$ ” S, $39^{\circ} 02^{\prime} 00$ ” W, altitude de $200 \mathrm{~m}$ e temperatura média anual de $25^{\circ} \mathrm{C}$. O clima do local, segundo a classificação de Thornthwaite, é do tipo úmido, com pluviosidade média anual de $1.170 \mathrm{~mm}$ e variações entre 900 e $1.300 \mathrm{~mm}$, sendo os meses mais chuvosos de março a agosto e os mais secos, de setembro a fevereiro (Ribeiro, 1998). O solo do local foi classificado como Argissolo Amarelo distrocoeso (Quadro 1), há cinco anos sob cultivo de laranja 'Pêra' (Citrus sinensis Osb.) sob porta-enxerto de limão-cravo (Citrus limonia Osbeck) em espaçamento de 5,0 m entre as linhas e 3,0 m entre plantas na linha, onde se plantou o feijão-de-porco (Canavalia ensiformis L.) como planta de cobertura do solo nas entrelinhas do pomar, durante os anos de 2006 e 2007. O feijão-de-porco foi plantado de duas formas: em 2006, em covas espaçadas de $0,2 \times 0,4 \mathrm{~m}$; em 2007, a lanço, utilizando-se aproximadamente $120 \mathrm{~kg}$ de sementes por hectare. Na implantação do pomar foram utilizadas uma aração e uma gradagem para preparar o solo e calagem e adubação para correção do $\mathrm{pH}$ e deficiências nutricionais, respectivamente. O plantio do citros foi feito em covas com dimensões de 0,4 x 0,4 x 0,3 m, adubadas com super-simples na dosagem média de $0,3 \mathrm{~kg}$ por cova. Para o cultivo e controle de plantas infestantes utilizaram-se grade e roçadeira nos dois primeiros anos e, a partir do terceiro ano, apenas roçadeira hidráulica tratorizada. A adubação foi feita com base em análise de solo, e os adubos, na formulação comercial 11-0808, foram distribuídos na quantidade de $0,4 \mathrm{~kg}$ por planta, de maneira uniforme na projeção da copa, normalmente entre os meses de maio e junho. Também se utilizou esterco curtido de frango, na quantidade de $4,0 \mathrm{~kg}$ por planta ano ${ }^{-1}$. A colheita, realizada duas vezes ao ano, nos meses de junho/julho e dezembro/janeiro, foi manual com carregamento via trator circulando nas entrelinhas das plantas. A produtividade média do pomar é de $10 \mathrm{t} \mathrm{ha}^{-1} \mathrm{ano}^{-1} \mathrm{de}$ frutos frescos.

\section{Avaliação de campo}

As medidas de resistência à penetração foram realizadas em duas profundidades do solo, $0-0,20$ e 0,20-0,40 $\mathrm{m}$, no período compreendido entre junho e dezembro de 2008. Para isso, estabeleceu-se na região central da entrelinha das plantas um transecto com $48 \mathrm{~m}$ de comprimento; neste, a cada $12 \mathrm{~m}$, identificouse um sítio de avaliação, totalizando cinco pontos (repetições) permanentes, nos quais as avaliações foram realizadas durante o período do estudo.

\section{Resistência à penetração}

O equipamento utilizado para medir resistência à penetração vertical foi o penetrômetro de impacto modelo comercial IAA/PLANALSUCAR/Stolf, segundo método descrito por Stolf et al. (1983). O penetrômetro de cone, também denominado de penetrômetro dinâmico, é um equipamento largamente utilizado em aplicações agrícolas para detectar camadas compactadas de solo. Apresenta como vantagens facilidade e velocidade de uso, baixo custo e forte relação com as condições físicas do solo para o crescimento do sistema radicular das plantas, além da possibilidade de medidas diretas de campo com grande número de repetições (Tormena \& Roloff, 1996; Herrick \& Jones, 2002; Naime et al., 2006). Sua desvantagem é a dependência de outros atributos do solo, a exemplo da umidade no momento da medida, textura, densidade do solo, estrutura, matéria orgânica, presença de pedras, mineralogia da argila e sistemas de uso e manejo (Gerard et al., 1982; Neiro et al., 2003).

Os resultados obtidos em impactos $\mathrm{dm}^{-1}$ foram convertidos em resistência dinâmica por meio da fórmula (Stolf, 1991):

$$
\mathrm{RP}=5,6+6,89(\mathrm{~N})
$$

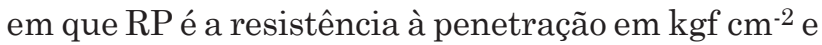
$\mathrm{N}$ é o número de impactos do peso metálico. Os resultados foram obtidos em $\mathrm{kgf} \mathrm{cm}^{-2}$ e depois multiplicados pela constante 0,0980665 para transformação em MPa, conforme Beutler et al. (2001). Após cada medida de resistência à penetração (RP) e nas mesmas profundidades, coletaram-se amostras deformadas de solo para determinação da umidade gravimétrica (Ug), conforme Embrapa (1997).

Quadro 1. Composição granulométrica para duas profundidades de um Argissolo Amarelo distrocoeso cultivado com citros e feijão-deporco como planta de cobertura do solo

\begin{tabular}{crrrr} 
Profundidade & Areia & Silte Argila & Classe Textural \\
\hline m & \multicolumn{3}{c}{$\mathrm{g} \mathrm{kg}^{-1}$} & \\
\cline { 2 - 4 } $0,00-0,20$ & 809 & 56 & 135 & Franco-arenosa \\
$0,20-0,40$ & 789 & 31 & 180 & Franco-arenosa \\
\hline
\end{tabular}


Objetivando avaliar a dinâmica da resistência à penetração durante o processo de secamento, estimouse para os modelos testados o valor da RP para valores de tensão da água no solo correspondentes: (a) 0,01 MPa, capacidade de campo; (b) 0,08 MPa, limite de sensibilidade para uso de tensiômetros no campo (Libardi, 2000); (c) 0,10 MPa, umidade crítica de manifestação do valor crítico de $2,0 \mathrm{MPa}$ para a maioria dos solos (Giarola et al., 2001); e (d) 1,5 MPa, ponto de murcha permanente. Para esse cálculo, o valor da umidade correspondente a cada tensão foi obtido na curva de retenção da água no solo. Essa curva foi determinada quantificando-se a água retida em amostras indeformadas de solo em uma mesa de tensão para as baixas tensões $(0,1,2,4$ e $6 \mathrm{kPa})$ e câmara de pressão de Richards para as altas tensões (30, 100, 300 e $1.500 \mathrm{kPa})$, conforme Embrapa (1997). Os pares de dados de umidade versus tensão foram ajustados à equação de van Genuchten (1980), utilizando-se o software RETC (van Genuchten et al., 2003).

\section{Estatística descritiva, análise exploratória dos dados e ajuste dos modelos}

Os valores de RP foram submetidos à análise estatística descritiva exploratória. Para isso, utilizouse o programa Statistica for Windows (Stat Soft, 1993). As seguintes medidas estatísticas foram feitas: média, mediana, moda, desvio-padrão, coeficiente de variação, valor mínimo, valor máximo, amplitude total, primeiro quartil, terceiro quartil, amplitude interquartil, assimetria e curtose, além da identificação de valores extremos, segundo as indicações de Libardi et al. (1996). A verificação da normalidade da distribuição dos dados foi feita com base nos coeficientes de assimetria e curtose, análise visual da reta de Henry e box-plot e confirmada pela aplicação do teste de Kolmogorov-Smirnov (K-S).

Os conjuntos de pares de dados foram plotados em um gráfico de RP x Ug (unidade gravimétrica), e equações de regressão foram ajustadas (Busscher et al., 1997), utilizando-se o protocolo definido pelo software Table Curve 2D, conforme Jandel (1991), escolhendo-se os modelos que apresentaram maior coeficiente de determinação.

\section{RESULTADOS E DISCUSSÃO}

Os parâmetros da estatística descritiva para a resistência à penetração do Argissolo Amarelo distrocoeso (Quadro 2) apresentaram diferenças importantes entre as profundidades avaliadas, porém nos dois casos os valores médios foram muito altos (Arshad et al., 1996) e ficaram muito acima do limite crítico de 2,5 MPa (Camargo \& Alleoni, 1997), indicando que as plantas cítricas do pomar avaliado encontram limitações físicas ao crescimento do sistema radicular durante parte significativa do ano. No entanto, deve-se considerar que a resistência à penetração possui relação potencial com a umidade do solo (Busscher et al., 1997), o que pode explicar os valores encontrados para a amplitude e o coeficiente de variação, tendo em vista que pequenas alterações no conteúdo de água determinam grandes variações na grandeza dependente, ou seja, na resistência à penetração. Nesse solo, esse comportamento decorre, possivelmente, da influência da textura do solo, cuja predominância da fração areia nas profundidades avaliadas (Quadro 1) resulta em rápida permeabilidade e consequente variação no conteúdo de água no solo, transferindo essa característica para a resistência à penetração.

Os valores da assimetria e curtose sugerem que a distribuição da resistência à penetração não é normal. A normalidade da distribuição é a premissa básica para a aplicação da maioria dos testes de significância e das técnicas de correlação e regressão (Ivo \& Fonteles Filho, 1997), o que determina a necessidade de atenção quanto a esse aspecto quando se comparam ou modelam dados dessa natureza. Indica também a validade da média como medida de tendência central de um conjunto de dados.

A tendência indicada pelos valores de assimetria e curtose quanto à distribuição pode ser confirmada pelos histogramas de frequência, reta de probabilidade de Henry (Figura 1), assim como pelo teste de Kolmogorov-Smirnov (K-S), cujos p-valor nas duas profundidades (Quadro 2) indicaram que a distribuição não é normal, o que também confirma os resultados da avaliação visual e do critério de similaridade entre média, moda e mediana. Percebe-se também que a

Quadro 2. Resumo da estatística descritiva para a resistência do solo à penetração em duas profundidades em um Argissolo Amarelo distrocoeso cultivado com citros e feijão-de-porco como planta de cobertura do solo

\begin{tabular}{|c|c|c|c|c|c|c|c|c|c|c|}
\hline Profundidade & $\mathbf{n}$ & Média & Mediana & Valor mínimo & Valor máximo & Amplitude & CV & Assimetria & Curtose & Teste K-S \\
\hline $\mathrm{m}$ & & & & $-\mathrm{MPa}$ & & - & $\%$ & & & \\
\hline $0,00-0,20$ & 30 & 5,07 & 2,31 & 1,01 & 26,17 & 25,16 & 120,48 & 2,11 & 4,31 & $\mathrm{p}<0,005$ \\
\hline $0,20-0,40$ & 30 & 6,22 & 3,51 & 1,51 & 33,18 & 31,67 & 104,00 & 2,75 & 9,81 & $p<0,100$ \\
\hline
\end{tabular}


distribuição da RP é perturbada pelos valores mais elevados (Figura 1b,d), os quais, levando-se em conta as características da variável em estudo, não podem ser considerados como atípicos e desprezados quando se estuda o fenômeno. A consequência desse resultado é a impossibilidade de uso da média como valor representativo do conjunto de dados, implicando interpretação mais segmentada quando se pretende determinar valores médios de resistência à penetração em uma área agrícola - a exemplo de separação em período úmido e período seco. Seguindo esse critério, observa-se (Figura 2) que no período úmido (junho a setembro) os valores da resistência à penetração foram bem menores que nos meses mais secos (outubro a dezembro) nas duas profundidades avaliadas. A mesma tendência ocorreu em relação ao coeficiente de variação (CV), que foi de $29,93 \%$, em 0-0,20 m, e 48,32 \%, em 0,20-0,40 m, no período úmido e bem maior no período seco, quando os valores alcançaram 81,08 \%, em 0-0,20 m, e 77,81\%, em 0,20-0,40 m (Quadro 3). De acordo com a classificação de Mulla \& McBratney (2000), esses valores podem ser classificados como altos para os dois períodos, à exceção da profundidade de 0-0,20 m, cujo valor de CV indica baixa variação. A separação em períodos distintos também parece alterar a distribuição dos dados. Neste caso, verifica-se, tanto pelo critério de comparação dos valores da média e mediana quanto pelos valores de assimetria e curtose, que a distribuição da resistência à penetração apresenta tendência de aproximar-se do padrão normal, o que pode ser confirmado pelos $\mathrm{p}$ valor do teste K-S (Quadro 3).
A umidade altera a coesão entre as partículas do solo (Beltrame et al., 1981). Assim, quando o solo está seco ou apresenta baixo conteúdo de água, suas partículas apresentam-se mais próximas e difíceis de serem separadas por qualquer força externa, justificando os valores encontrados no mês de novembro. Em dezembro, verificou-se significativa redução dos valores de $\mathrm{RP}$ em relação a novembro, determinada pelo aumento da umidade do solo causado por uma chuva que ocorreu pouco antes da amostragem.

No quadro 4 podem ser observados os modelos e as respectivas equações e, nas figuras 3 e 4, os diagramas de dispersão para os modelos ajustados. Os valores permitem afirmar, conforme amplamente registrado em literatura, que a resistência à penetração foi significativamente relacionada com a umidade do solo, sendo negativamente influenciada por esta em todos os modelos testados, os quais se revelaram significativos (teste $\mathrm{F}_{\alpha=0,05}$ ) e representativos $\left(\mathrm{R}^{2}\right)$, sendo os exponenciais aqueles que mostraram os maiores valores de $\mathrm{R}^{2}$ nas duas profundidades. $\mathrm{A}$ correlação pode ser descrita como inversa moderada (Ivo \& Fonteles Filho, 1997), e o fato de todos os modelos apresentarem-se significativos dificulta a indicação de um deles como o ideal, mesmo porque o modelo exponencial difere daquele encontrado por Busscher et al. (1997), que, ao discutirem a correlação entre o índice de cone e o conteúdo de água no solo, verificaram que o modelo potencial foi o mais adequado para modelar essa relação. Observa-se também que este modelo tende a superestimar a resistência à penetração em relação ao exponencial, nas duas profundidades (Quadro 5). (a)

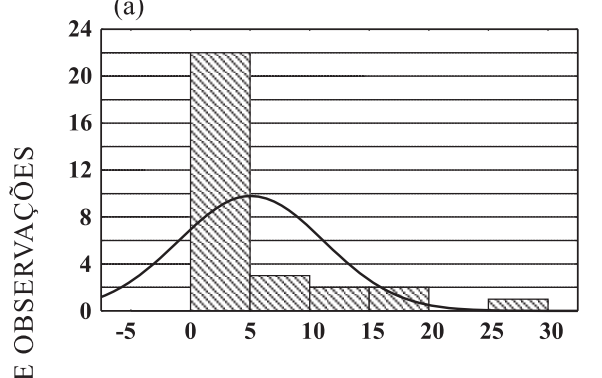

(c)

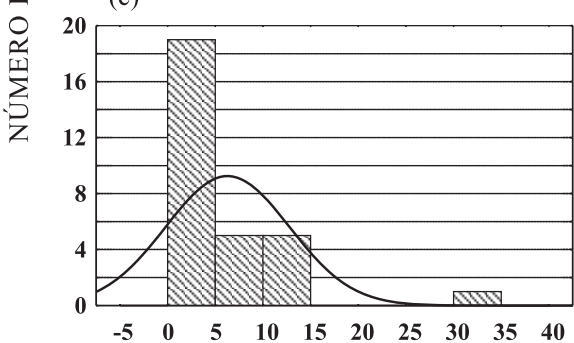

$\mathbf{0 , 0}-\mathbf{0 , 2 0} \mathrm{m}$

(b)

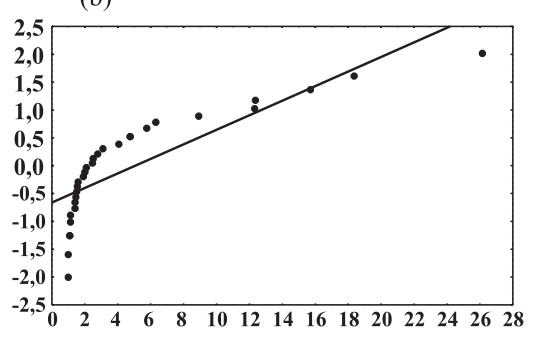

0,20-0,40 m

(d)

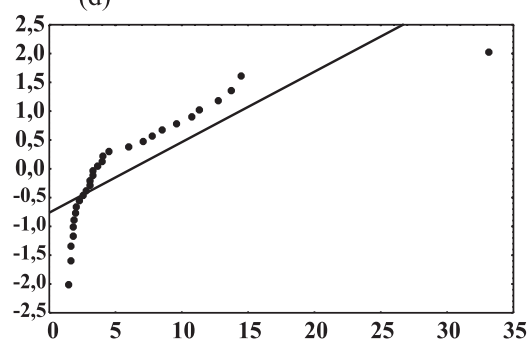

RESISTÊNCIA À PENETRAÇÃO

Figura 1. Histogramas de frequência $(a, c)$ e reta de probabilidade de Henry $(b, d)$ para a resistência à penetração de um Argissolo Amarelo distrocoeso cultivado com citros e feijão-de-porco como planta de cobertura do solo. 
Valores entre 2 e 2,5 MPa têm sido indicados como os limites críticos de resistência à penetração para $\mathrm{a}$ maioria dos vegetais. Assumindo o valor de 2,5 $\mathrm{MPa}$ como o limite crítico ao crescimento das raízes (Camargo \& Alleoni, 1997), pode-se, a partir dos modelos ajustados, fazer uma série de inferências sobre a dinâmica da resistência à penetração durante o processo de secamento do solo, desde a capacidade de campo até o ponto de murchamento. Os resultados dessas estimativas mostram que os diferentes modelos estimaram valores próximos para a RP para os quatro conteúdos de umidade considerados nessa simulação (Quadro 5). Outro aspecto importante é que, mesmo na capacidade de campo, o Argissolo Amarelo

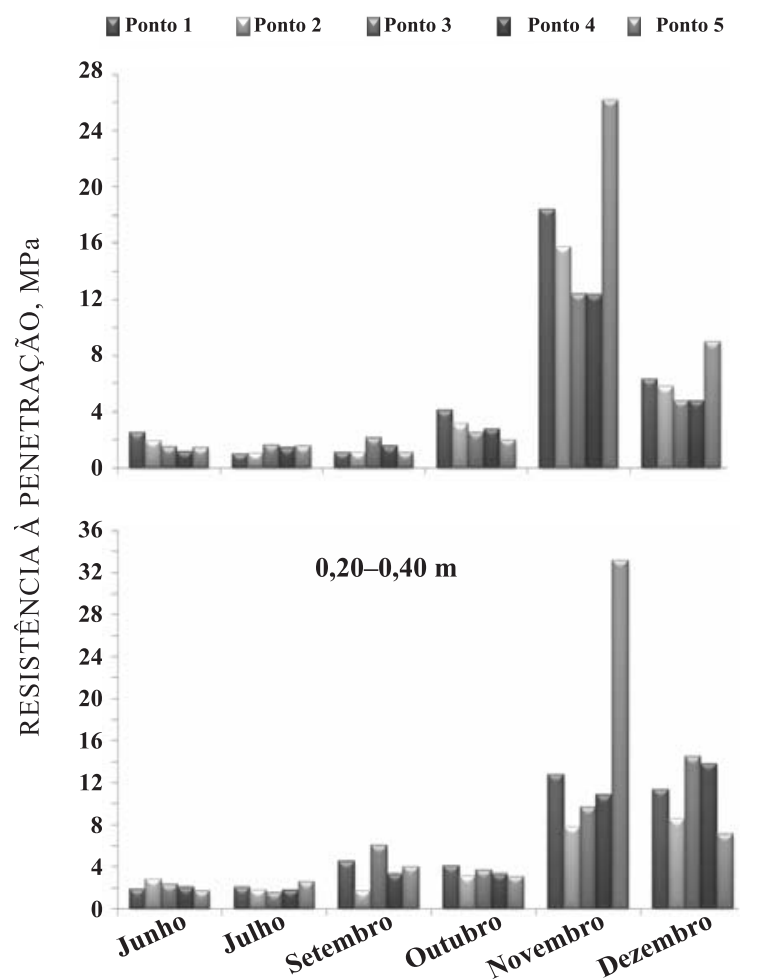

Figura 2. Variação temporal da resistência à penetração para um Argissolo Amarelo distrocoeso cultivado com citros e feijão-deporco como planta de cobertura do solo.
Quadro 4. Modelos matemáticos ajustados $(\alpha=0,05)$ para a relação entre resistência à penetração (MPa) e umidade no solo $\left(\mathrm{kg} \mathrm{kg}^{-1}\right)$ em um Argissolo Amarelo distrocoeso cultivado com citros e feijão-de-porco como planta de cobertura do solo, no Recôncavo da Bahia

\begin{tabular}{llrr}
\hline \multicolumn{1}{c}{ Modelo } & \multicolumn{1}{c}{ Equação } & $\mathbf{R}^{2}$ & $\mathbf{F}$ \\
\hline & \multicolumn{1}{c}{$0,0-0,20 \mathrm{~m}$} \\
Exponencial 1 & $R P=26,90 \exp ^{\frac{-U g}{0,024}}$ & 0,790 & 94,12 \\
Exponencial 2 & $R P=0,75+27,28 \exp ^{\frac{-U g}{0,022}}$ & 0,761 & 121,58 \\
Potencial 1 & $R P=-12,85+5,14 U g^{-0,392}$ & 0,750 & 36,10 \\
Potencial 2 & $R P=0,13 U g^{-1,098}$ & 0,689 & 55,59 \\
& $\quad 0,20-0,40 \mathrm{~m}$ & & \\
Exponencial 1 & $R P=25,47 \exp ^{\frac{-U g}{0,027}}$ & 0,740 & 142,65 \\
Exponencial 2 & $R P=0,97+27,52 \exp ^{\frac{-U g}{0,022}}$ & 0,750 & 73,69 \\
Potencial 1 & $R P=-7,22+2,42 U g^{-0,521}$ & 0,704 & 58,11 \\
Potencial 2 & $R P=0,17 U g^{-1,050}$ & 0,662 & 98,33 \\
\hline
\end{tabular}

distrocoeso apresenta valores de RP acima de 2,5 MPa, considerado alto por Arshad et al. (1996), o que resulta em algumas limitações ao crescimento das raízes das plantas (Camargo \& Alleoni, 1997) mesmo para conteúdos elevados de umidade do solo. Quando a tensão da água no solo atinge $0,08 \mathrm{MPa}$, limite da sensibilidade dos tensiômetros no campo, o valor da RP já é considerado muito alto (Arshad et al., 1996), proporcionando sérias limitações ao crescimento das raízes, mesmo quando o conteúdo de água no solo não é limitante. Essa realidade é acentuada pela rapidez com que esses solos perdem água, tendo em vista sua elevada permeabilidade (Silveira, 2009) e textura franco-arenosa, pouco eficiente na retenção de água (Quadro 1). Do ponto de vista prático, esses resultados são indicadores da necessidade de uso de técnicas para a manutenção da umidade no solo próxima à capacidade de campo como prática de manejo para o controle da resistência do solo à penetração e oferta de condições satisfatórias para o crescimento das plantas cítricas cultivadas em solos com essas características.

Quadro 3. Resumo da estatística descritiva para a resistência do solo à penetração em dois períodos do ano, em duas profundidades, em um Argissolo Amarelo distrocoeso cultivado com citros e feijão-de-porco como planta de cobertura do solo, no Recôncavo da Bahia

\begin{tabular}{|c|c|c|c|c|c|c|c|c|c|c|}
\hline Período & $\mathbf{n}$ & Média & Mediana & Valor mínimo & Valor máximo & Amplitude & CV & Assimetria & Curtose & Teste K-S \\
\hline & & & & $\mathrm{MPa}$ & 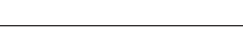 & & $\%$ & & & \\
\hline Jun a set & 15 & 1,47 & 1,43 & 1,01 & $\begin{array}{l}0,0-0,20 \mathrm{~m} \\
2,52\end{array}$ & 1,51 & 29,93 & 1,15 & 0,98 & $\mathrm{p}>0,20$ \\
\hline Out a dez & 15 & 8,67 & 5,80 & 2,01 & 26,17 & 24,16 & 81,08 & 1,32 & 1,26 & $\mathrm{p}>0,20$ \\
\hline Jun a set & 15 & 2,66 & 2,07 & 1,51 & $\begin{array}{l}0,20-0,40 \mathrm{~m} \\
6,03\end{array}$ & 4,52 & 48,49 & 1,59 & 2,60 & $\mathrm{p}>0,20$ \\
\hline Out a dez & 15 & 9,78 & 8,52 & 3,09 & 33,18 & 30,09 & 77,81 & 2,19 & 6,34 & $\mathrm{p}>0,20$ \\
\hline
\end{tabular}




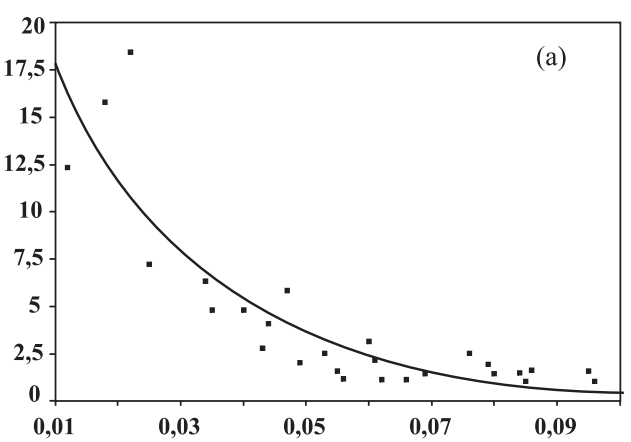

$\mathbf{0 , 0 - 0 , 2 0 ~ m ~}$
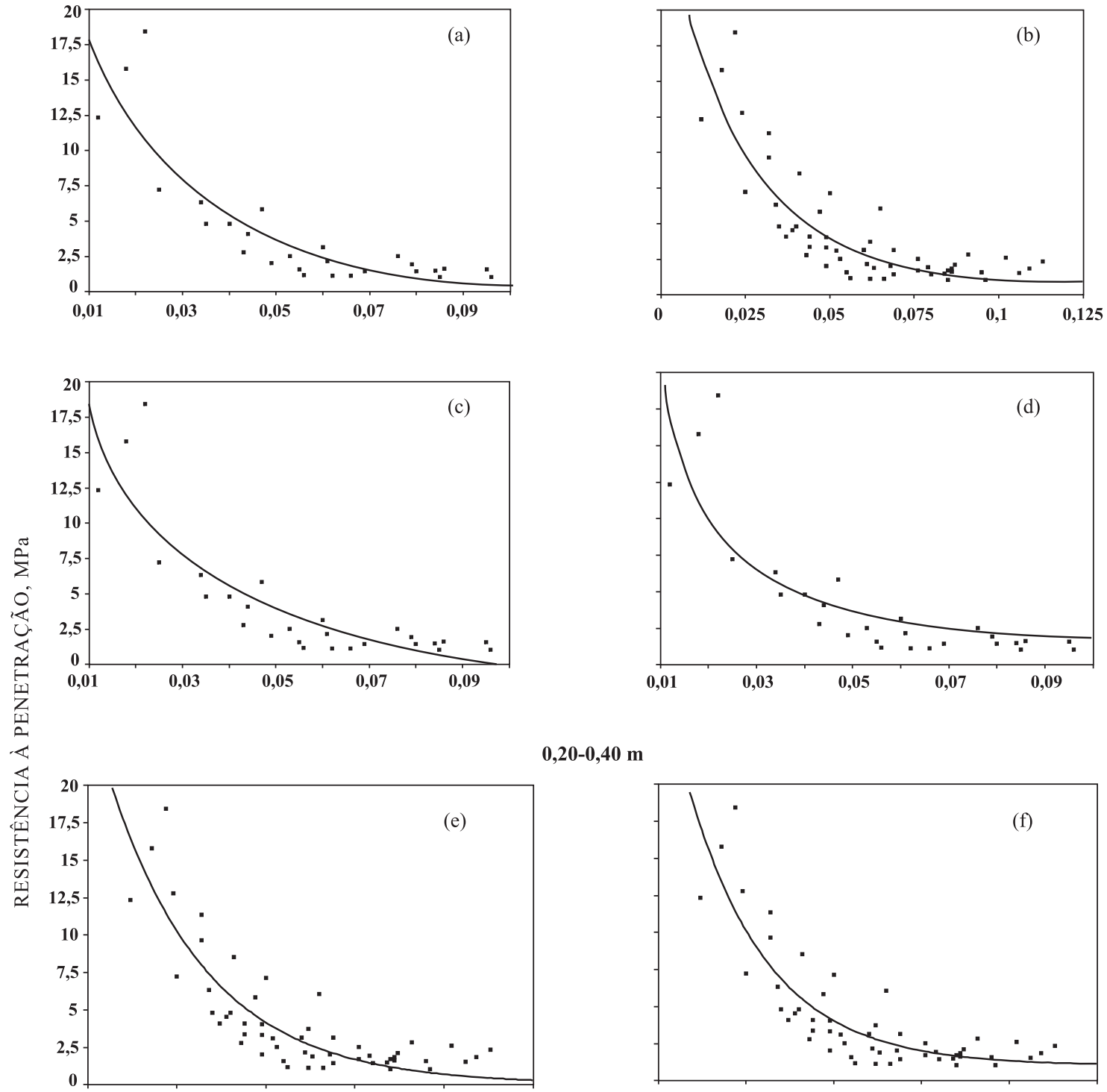

0,20-0,40 m
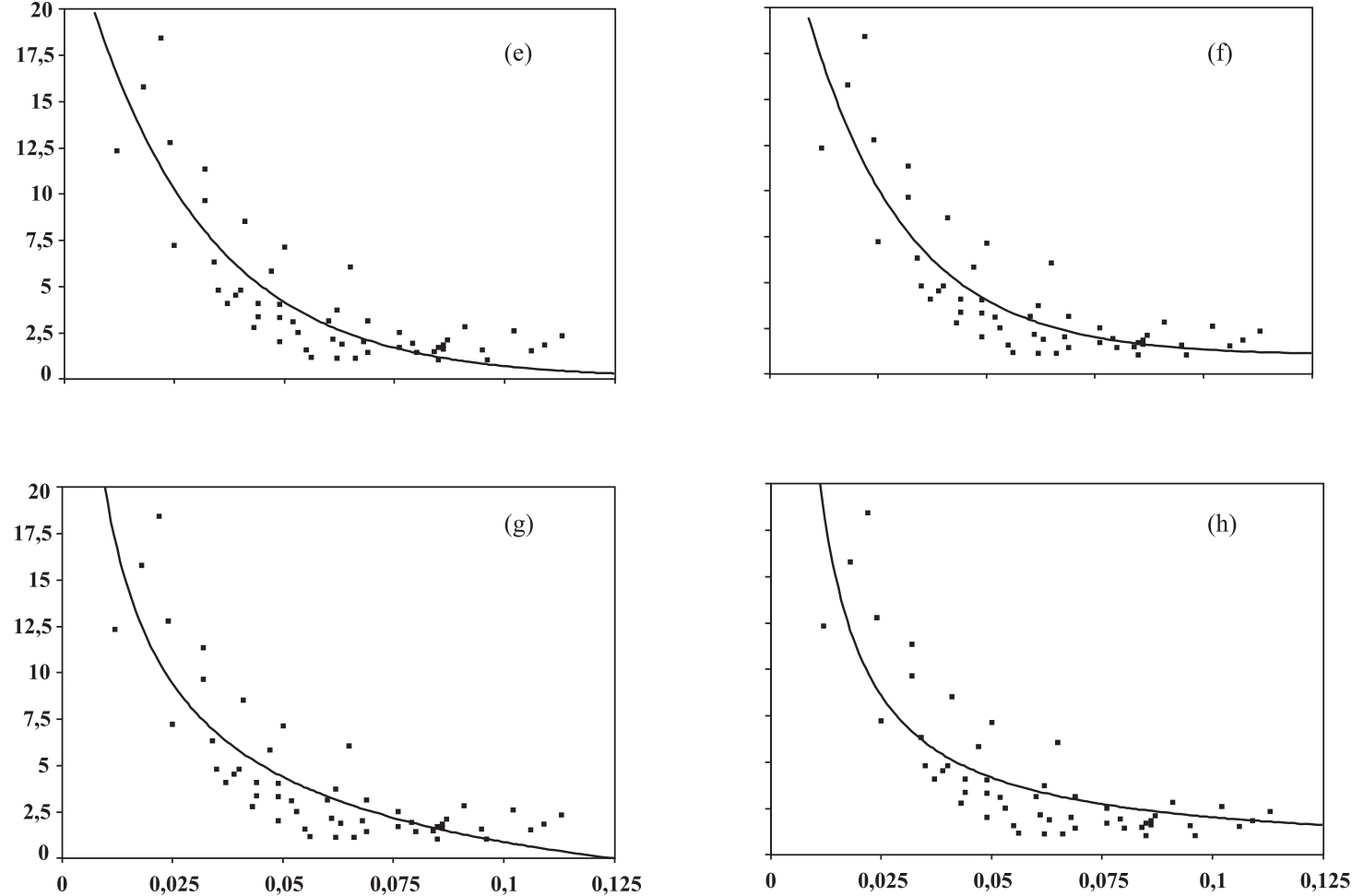

UMIDADE DO SOLO, $\mathrm{kg} \mathrm{kg}^{-1}$

Figura 3. Curvas de ajuste da relação umidade do solo versus resistência à penetração para as profundidades de 0,0-0,20 m de um Argissolo Amarelo e 0,20-0,40 m de um Argissolo Amarelo distrocoeso cultivados com citros e feijão-de-porco como planta de cobertura do solo, no Recôncavo da Bahia. (a) Exponencial 1; (b) Exponencial 2; (c) Potencial 1; (d) Potencial 2; (e) Exponencial 1; (f) Exponencial 2; (g) Potencial 1; e (h) Potencial 2. 
Quadro 5. Valores de referência para resistência à penetração em quatro níveis de tensão da água no solo segundo quatro modelos matemáticos de ajuste da relação umidade $\left(\mathrm{kg} \mathrm{kg}^{-1}\right)$ versus resistência à penetração em um Argissolo Amarelo distrocoeso cultivado com citros e feijão-de-porco como planta de cobertura do solo

\begin{tabular}{|c|c|c|c|c|}
\hline \multirow{2}{*}{ Modelo } & \multicolumn{4}{|c|}{ Tensão da água no solo (MPa) } \\
\hline & 0,01 & 0,08 & 0,10 & 1,5 \\
\hline & \multicolumn{4}{|c|}{$0,0-0,20 \mathrm{~m}$} \\
\hline$R P=26,90 \exp ^{\frac{-U g}{0,024}}$ & 2,25 & 4,41 & 4,43 & 4,47 \\
\hline$R P=0,75+27,28 \exp \frac{-\frac{-g}{0,022}}{0.04}$ & 2,58 & 4,54 & 4,56 & 4,60 \\
\hline$R P=-12,85+5,14 U g^{-0,392}$ & 2,69 & 4,73 & 4,75 & 4,78 \\
\hline \multirow[t]{2}{*}{$R P=0,13 U g^{-1,098}$} & 2,88 & 4,07 & 4,08 & 4,10 \\
\hline & \multicolumn{4}{|c|}{$0,20-0,40 \mathrm{~m}$} \\
\hline$R P=25,47 \exp \frac{-U g}{0,027}$ & 2,42 & 3,55 & 3,58 & 5,30 \\
\hline$R P=0,97+27,52 \exp ^{\frac{-U g}{0,022}}$ & 2,50 & 3,42 & 3,44 & 5,38 \\
\hline$R P=-7,22+2,42 U g^{-0,521}$ & 2,95 & 3,94 & 3,96 & 5,48 \\
\hline$R P=0,17 U g^{-1,050}$ & 3,07 & 3,70 & 3,72 & 4,58 \\
\hline
\end{tabular}

\section{CONCLUSÕES}

1. A resistência à penetração do Argissolo Amarelo distrocoeso apresentou distribuição diferente da normal, elevado coeficiente de variação e elevada correlação negativa com a umidade do solo.

2. Os modelos testados mostraram bom ajuste e foram adequados para descrever a relação entre umidade e resistência à penetração do solo, sendo o modelo exponencial o que apresentou os melhores índices de aferição para essa relação.

\section{LITERATURA CITADA}

ARSHAD, M.A.; LOWERY, B. \& GROSSMAN, B. Physical tests for monitoring soil quality. In: DORAN, J.W. \& JONES, A.J., eds. Methods for assessing of soil quality. Madison, Soil Science Society of American/American Society of Agronomy, 1996. p.123-141. (SSSA Special Publication, 49)

BEUTLER, A.N.; SILVA, M.L.N.; CURI, N.; FERREIRA, M.M.; CRUZ, J.C. \& PEREIRA FILHO, I.A. Resistência à penetração e permeabilidade de Latossolo Vermelho distrófico típico sob sistemas de manejo na região dos cerrados. R. Bras. Ci. Solo, 25:167-177, 2001.

BELTRAME, L.F.S.; GONDIN, L.A.P. \& TAYLOR, J.C. Estrutura e compactação na permeabilidade de solos do Rio Grande do Sul. R. Bras. Ci. Solo, 5:145-149, 1981.

BUSSCHER, W.J.; BAUER, P.J.; CAMP, C.R. \& SOJKA, R.E. Correction of cone index for soil water content differences in a coastal plain soil. Soil Till. Res., 43:205-217, 1997.
CAMARGO, O.A. \& ALLEONI, L.R.F. Compactação do solo e o desenvolvimento de plantas. Piracicaba, Escola Superior de Agricultura Luiz de Queiroz, 1997. 132p.

CARVALHO, J.E.B.; PAES, J.M.V. \& MENEGUCCI, J.L.P. Manejo de plantas daninhas em citros. Inf. Agropec., 22:61$70,2001$.

CINTRA, F.L.D.; LIBARDI, P.L. \& SILVA, A.P. Tabuleiros costeiros do Nordeste do Brasil: Uma análise dos efeitos do regime hídrico e da presença de camadas coesas dos solos. B. Inf. SBCS., 18:81-95, 1997.

CINTRA, F.L.D.; LIBARDI, P.L. \& JORGE, L.A.C. Distribuição do sistema radicular de porta-enxertos de citros em ecossistema de Tabuleiro Costeiro. R. Bras. Frutic., 21:313317,1999

EMPRESA BRASILEIRA DE PESQUISA AGROPECUÁRIA EMBRAPA. Centro Nacional de Pesquisa de Solo. Manual de métodos de análise de solo. Rio de Janeiro, 1997. 212p.

EMPRESA BRASILEIRA DE PESQUISA AGROPECUÁRIA EMBRAPA. Centro Nacional de Pesquisa de Solo. Sistema brasileiro de classificação de solos. Rio de Janeiro, 2006. 306p.

FERNANDES, J.C.; RODRIGUES, J.G.L.; GAMERO, C.A.; ACOSTA, J.J.B. \& LANCAS, K.P. Resistência do solo à penetração em diferentes sistemas de manejo e velocidades de semeadura no desenvolvimento de um cultivar de triticale. Energ. Agric., 22:55-64, 2007.

GERARD, C.J.; SEXTON, P. \& SHAW, G. Physical factors influencing soil strength and root growth. Agron. J., 74:875-879, 1982.

GIAROLA, N.F.B.; SILVA, A.P.; TORMENA, C.; SOUZA, L.S. \& RIBEIRO, L.P. Similaridades entre o caráter coeso dos solos e o comportamento Hardsetting: Estudo de caso. R. Bras. Ci. Solo, 25:239-247, 2001. 
HERRICK, J.E. \& JONES, T.L. A dynamic cone penetrometer for measuring soil penetration resistance. Soil Sci. Soc. Am. J., 66:1320-1324, 2002.

IMHOFF, S.; SILVA, A.P. \& TORMENA, C.A. Aplicações da curva de resistência no controle da qualidade física de um solo sob pastagem. Pesq. Agropec. Bras., 35:1403-1500, 2000 .

IVO, C.T.C. \& FONTELES FILHO, A.A. Estatística pesqueira: Aplicações em engenharia de pesca. Fortaleza, Tom Gráfica, 1997.193p.

JANDEL SCIENTIFIC. Table Curve: Curve fitting software. Corte Madera, 1991. 280p.

LIBARDI, P.L. Dinâmica da água no solo. 2.ed. Piracicaba, O Autor, 2000. 509p.

LIBARDI, P.L. \& MELO FILHO, J.F. Influência dos horizontes coesos na dinâmica da água no solo. In: WORKSHOP COESÃO EM SOLOS DOS TABULEIROS COSTEIROS, 2001, Aracaju. Coesão em solos dos Tabuleiros Costeiros. Aracaju, Embrapa Tabuleiros Costeiros, 2001. p.193-208.

LIBARDI, P.L.; MANFRON, P.A.; MORAES, S.O. \& TUON, R.L. Variabilidade da umidade gravimétrica de um solo hidromórfico. R. Bras. Ci. Solo, 20:1-12, 1996.

MERCANTE, E.; URIBE-OPAZO, M.A. \& SOUZA, E.G. Variabilidade espacial e temporal da resistência mecânica do solo à penetração em áreas com e sem manejo químico localizado. R. Bras. Ci. Solo, 27:1149-1159, 2003.

MULLA, D.J. \& McBRARNEY, A.B. Soil spatial variability. In: SUMNER, M.E., ed. Handbook of soil science. New York, CRC Press, 2000. p.321-352.

MÜLLER, M.M.L.; CECCON, G. \& ROSOLEM, C.A. Influência da compactação do solo em subsuperfície sobre o crescimento aéreo e radicular de plantas de adubação verde de inverno. R. Bras. Ci. Solo, 25:531-538, 2001.

NAIME, J.M.; JUNIOR. J.F.; RABELO, L.M. \& VAZ, C.M.P. Penetrômetro dinâmico automatizado - Versão eletrônica. São Carlos, Embrapa Instrumentação Agropecuária, 2006. (Boletim Técnico, 79)

NEIRO, E.S.; MATA, J.D.V.; TORMENA, C.A.; GONÇALVES, A.C.A.; PINTRO, J.C. \& COSTA, J.M. Resistência à penetração de um Latossolo Vermelho distroférrico, com rotação e sucessão de culturas, sob plantio direto. Acta Sci. Agron., 25:19-25, 2003.

PATRIZZI, V.C.; VAZ, C.M.P.; IOSSI, M.F. \& PRIMAVESI, O. Modelamento do efeito da umidade e densidade na resistência à penetração dos solos. In: CONGRESSO BRASILEIRO DE CIÊNCIA DO SOLO, 29., Ribeirão Preto, 2003. Anais. Ribeirão Preto, SBCS/UNESP, 2003. CDROM.

Pedrotti, A.; PAUletto, E.A. \& CRESTANA, S. Resistência mecânica à penetração de um Planossolo submetido a diferentes sistemas de cultivo. R. Bras. Ci. Solo, 25:521-529, 2001.
PEREIRA, J.O.; SIQUEIRA, J.A.C.; URIBE-OPAZO, M.A. \& SILVA, S.L. Resistência do solo à penetração em função do sistema de cultivo e teor de água do solo. R. Bras. Eng. Agríc. Amb., 6:171-174, 2002.

REZENDE, J.O. Solos coesos dos Tabuleiros Costeiros: Limitações agrícolas e manejo. Salvador, SEAGRI-SPA, 2000. 117p. (Série Estudos Agrícolas, 1)

REYNOLDS, W.D.; BOWMAN, B.T.; DRURY, C.F.; TAN, C.S. \& LU, X. Indicators of good soil physical qualify: Density and storage parameters. Geoderma, 110:131-146, 2002.

RIBEIRO, L.P. Os Latossolos Amarelos do Recôncavo Baiano: Gênese, evolução e degradação. Salvador, Seplantec, 1998. $99 \mathrm{p}$.

SANTANA, M.B. Atributos físicos do solo e distribuição do sistema radicular de citros como indicadores da coesão em dois solos dos Tabuleiros Costeiros do Estado da Bahia. Viçosa, MG, Universidade Federal de Viçosa, 2003. 76p. (Tese de Mestrado)

SILVEIRA, D.C. Diagnóstico da qualidade do solo em um pomar de citros cultivado com plantas de cobertura nas condições ambientais do Recôncavo da Bahia. Cruz das Almas, Universidade Federal do Recôncavo da Bahia, 2009. 73p. (Tese de Mestrado)

SOUZA, L.S.; SOUZA, L.D.; PAIVA, A.Q.; RODRIGUES, A.C.V. \& RIBEIRO, L.S. Distribuição do sistema radicular de citros em uma topossequência de solos de Tabuleiro Costeiro do Estado da Bahia. R. Bras. Ci. Solo, 32:503513,2008 .

STAT SOFT. Statistica for Windows [Eletronic manual index], v.4.3. Tulsa, 1993. $2 \mathrm{v}$.

STOLF, R.; FERNANDES, J. \& URLANI NETO, V.L. Recomendação para o uso do penetrômetro de impacto modelo IAA/Planalsucar - Stolf. São Paulo, MIC/IAA/ PNMCA-Planalsucar, 1983. 8p. (Boletim, 1)

STOLF, R. Teoria e teste experimental de fórmulas de transformação dos dados de penetrômetro de impacto em resistência do solo. R. Bras. Ci. Solo, 15:229-235, 1991.

TORMENA, C.A. \& ROLOFF, G. Dinâmica da resistência à penetração de um solo sob plantio direto. R. Bras. Ci. Solo, 20:333-339, 1996.

TORMENA, C.A.; BARBOSA, M.C. \& COSTA, A.C.S. Densidade, porosidade e resistência à penetração em Latossolo cultivado sob diferentes sistemas de preparo do solo. Sci. Agríc., 59:795-801, 2002.

van GENUCHTEN, M.T. A closed form equation for predicting hydraulic conductivity of unsaturated soils. Soil Sci. Soc. Am. J., 44:892-898, 1980.

van GENUCHTEN, M.T.; SINUNEK, J.; LEIJ, F.J. \& SEGMA, M. Code for quantifying the hydraulic functions of unsatured soils. Riverside, US Salinity Laboratory, USDA, ARS, 2003. 